\title{
Steering simulation performance in patients with obstructive sleep apnoea and matched control subjects
}

\author{
M. Juniper*, M.A. Hack*, C.F. George**, R.J.O. Davies*, J.R. Stradling*
}

\begin{abstract}
Steering simulation performance in patients with obstructive sleep apnoea and matched control subjects. M. Juniper, M.A. Hack, C.F. George, R.J.O. Davies, J.R. Stradling. C)ERS Journals Ltd 2000.

ABSTRACT: Patients with obstructive pulmonary disease (OSA) have an increased rate of driving accidents, perhaps due to poor vigilance or impaired cognitive skills that influence their driving ability. The authors have assessed whether patients with OSA perform differently to control subjects on a steering simulator which allows the separate assessment of the two visual tasks required for steering a car, immediate positioning on road with reference to the road edges, and assessment of the curve of the oncoming road which allows faster driving.

Twelve patients with OSA and 12 control subjects, matched for age, sex and driving experience, performed three 30-min drives with either all the oncoming road visible, only the near part of the road visible, or only the distant part of the road visible. Steering was assessed by measuring the SD around the theoretical perfect path (steering error) and the number of times the driver went "off road". Subjects identified the appearance of target numbers at the four corners of the screen as quickly as possible, thus making the test a divided attention task.

Patients with OSA performed significantly less well on the three different road fields as measured by steering error $(p<0.001)$, time to detect the target number $(p<0.03)$, and off road events $(p<0.03)$. The patients appeared to be particularly impaired on the two drives when only part of the road ahead was available to guide steering.

This steering simulator, with its more realistic view of the road ahead, identifies impaired performance in patients with obstructive sleep apnoea. In addition it suggests that patients with obstructive sleep apnoea may be more disadvantaged compared to normal subjects when the view of the road ahead is limited (such as in fog). Eur Respir J 2000; 15: 590-595.
\end{abstract}

* Osler Chest Unit, Churchill Hospital, Oxford, UK. **Sleep Laboratory, Victoria Hospital, London, Ontario, Canada.

Correspondence: J. Stradling

Osler Chest Unit

Churchill Hospital

Oxford

OX3 7LJ

UK

Fax: 441865225221

Keywords: Automobile accidents car accidents

driving

obstructive sleep apnoea

steering simulation

Received: June 231999

Accepted after revision December 41999

This work was supported by the British Lung Foundation
Driving a car is a complex task involving the interpretation of incoming visual, auditory and tactile stimuli to produce the necessary motor responses. Two distinct visual tasks are involved in steering accurately along a road [1]. The first involves processing the view of the road close to the vehicle which enables the driver to maintain exact "position in lane" by alignment of the vehicle relative to the kerb. The second involves the view of the distant road, which allows the driver to predict the required position of the car by anticipating the curve of the road ahead, and thus allows faster driving. This also allows a driver, who has a good view ahead, to look briefly away from the road and maintain the position of the vehicle on the road.

Many factors, for example alcohol consumption, sleep deprivation, certain drugs and driving experience will influence the ability to steer a vehicle, and it is known that patients with untreated obstructive sleep apnoea (OSA) have an increased risk of motor vehicle accidents [2-13]. In patients with OSA the repeated episodes of pharyngeal collapse at sleep onset cause recurrent arousal from sleep. This disrupts sleep architecture and leads to day-time somnolence and cognitive impairment $[14,15]$. It is not clear what aspect of OSA causes patients to have diffi- culty in driving, and in particular whether it is simply due to reduced vigilance or other factors, such as cognitive impairment, that could reduce the ability to process accurately the incoming information.

It would be unethical to test patients with OSA in a "real" car on a "real" road, therefore simulation has become an accepted way of investigating driving performance, although simulators cannot reproduce all aspects of the task of driving.

Driving performance on simple simulators has been shown to be impaired in OSA and improves in uncontrolled studies following treatment with nasal continuous positive airway pressure (nCPAP) [12, 16-18]. These simulations have measured vigilance, reaction time, visual search and measures of tracking. They have not included a realistic perspective view of a road curving ahead and have therefore not examined both of the visual tasks of driving mentioned previously.

The authors have therefore compared the steering performance of 12 patients with moderately severe OSA with 12 healthy matched control subjects. The authors have assessed the learning time required to perform the steering task and the reproducibility of the test results. 


\section{Methods}

\section{Subjects}

Twelve patients with untreated OSA, with $\geq 10 \cdot \mathrm{h}^{-1}$ of $>4 \%$ oxygen saturation dips, and an Epworth Sleepiness Score (ESS) $\geq 10$, were recruited through the outpatient department of the Oxford Sleep Unit following a diagnostic sleep study. Twelve control subjects without OSA were recruited having had normal sleep studies as part of a large community survey of the prevalence of OSA, recently completed in the authors' unit. The 12 control subjects and 12 patients with OSA were matched for median age (48.5 (5th/95th centiles; 38/62), 48.0 (37/63) yrs respectively; $\mathrm{p}=0.9)$. All subjects were male, had held a current UK driving licence for $>10$ yrs, and drove $>250$ $\mathrm{h} \cdot \mathrm{yr}^{-1}$. Results of sleep studies were normal in the control group, compared with patients ( $>4 \%$ arterial oxygen saturation $\left(\mathrm{Sa}, \mathrm{O}_{2}\right)$ median dip rate per hour; $0.8(0 / 2.9)$ and 41.1 $(16 / 61)$ respectively; $p<0.0001)$ and the control subjects had no increased subjective daytime sleepiness, compared with patients, measured by median ESS (6.5 (2.6/9.9), 16.5 $(12.6 / 20.8)$ respectively; $p<0.0001)$.

Thirty healthy male subjects with no history of snoring or symptoms of OSA were recruited for a study on the "learning effect" when using this driving simulator. The thirty subjects recruited had a median age of 31 yrs (22/ 71); body mass index (BMI), 23.5 (18/32); ESS, 6 (1/9); licence held, 13.5 yrs (3/35); hours driven per year, 343 (39/1596).

Fourteen healthy male subjects with the same entry requirements were recruited for a study on the reproducibility of the results obtained from this driving simulator on consecutive days. For this short term reproducibility study (1 day) the subjects recruited had a median age of 27.5 yrs (23/51); BMI, 24.3 (22/28); ESS, 5 (2/7); licence held, 8 yrs (4/29); hours driven per year, 240 (38/553). Twelve similar subjects were recruited for the longer term reproducibility with a median age of 21 yrs (20/46); BMI, 22 (17/34); ESS, 6 (1/12); licence held, 3 yrs (2/13); hours driven per year, $145(56 / 360)$; time interval between tests, 61 days $(3 / 142)$.

\section{Diagnostic sleep studies}

Prior to selection, all patients with OSA underwent the authors' standard in-hospital one night sleep study (Visilab; Stowood Scientific Instruments, Oxford, UK). The presence of OSA was established by recording body movement and heart rate as markers of sleep disturbance, with $\mathrm{Sa}_{\mathrm{a}} \mathrm{O}_{2}$ and snoring as markers of respiratory impairment [19]. A video recording of the whole night was available to confirm that the abnormalities seen were due to upper airway obstructive events. The severity of OSA was quantified from the number of $>4 \%$ falls in $\mathrm{Sa}_{2} \mathrm{O}_{2}$ per hour of study. This index predicts the severity of OSA symptoms and its response to nCPAP treatment at least as well as other commonly used indices [19].

The twelve control subjects had home studies of oximetry, body position, snoring, thoracic movement and pulse transit time (RM50; Parametric Recorders, London, UK) as part of a community survey which confirmed the absence of OSA. The pulse transit time provided information on inspiratory effort [20] and autonomic arousals $[19,21]$. All studies were analysed by standard procedures used in the authors' unit.

\section{Steering simulation}

A steering simulator was developed based on the work of LAND and Horwood [1]. The program was written in Q Basic. A computer-based image of the moving edges of a pseudorandomly winding road are portrayed white on black as in night driving. An image of the bonnet of the vehicle is portrayed at the bottom of the screen. The subject steers using a standard computer games steering wheel (Grandprix 1; Thrustmaster, Hillboro, OR, USA). The object of each test is to steer the centre of the vehicle as accurately as possible down the centre of the road. The correct and realistic relationship between turning the wheel and the effect this has on car position has been carefully established [1]. Each test is a maximum of 30 min duration and either the whole road ("all"), the near field ("near") or the distant field ("far") are visible to the driver to aid positioning of the car (fig. 1).

Single digits, $1-9$, which randomly change at $\sim 8-10 \mathrm{~s}$ intervals, are displayed at each corner of the computer screen. While steering, the subject is required to scan the four corners and identify a target digit each time it appears, approximately once per minute, by pressing a button on either side of the steering wheel. By including this visual search task a divided attention task is produced. Prior to commencing their training, all subjects received the same written and verbal instructions on the use of the simulator and the aim of the test.

The initial $60 \mathrm{~s}$ of each test were excluded from the analysis to avoid including any "settling time". Position on the road relative to the centre was measured twenty times per second. The SD of this data was used to give a measure of the steering error during the simulated drive. Mean
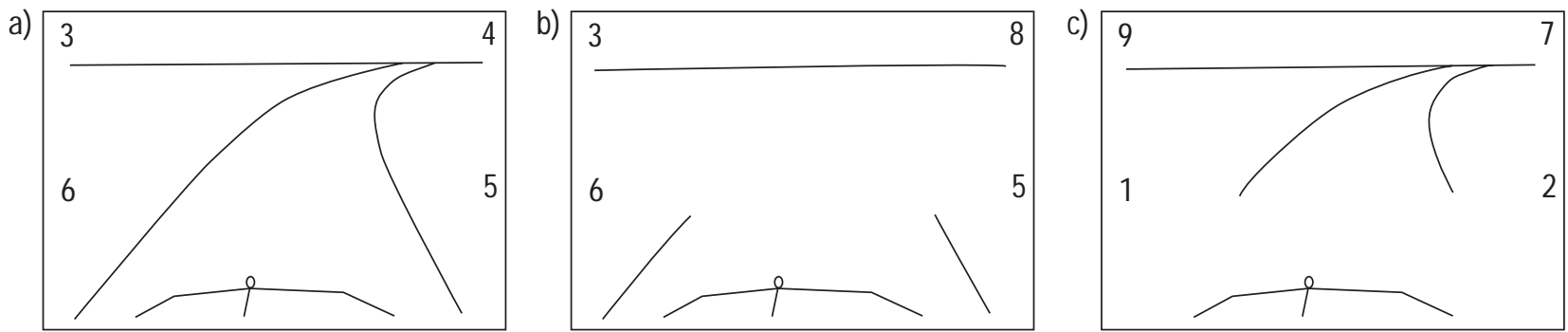

Fig. 1. - Example of the three different roads visible during simulated drives, a) "all" the road, b) "near" field only, and c) "far" field only. 
reaction time for the detection of target digits was also measured. The authors also counted "off road" events which were defined as occurring when the centre of the vehicle's bonnet crossed either lateral border of the road. The number of these events was quoted per hour of driving to allow for different length drives. The driver "crashed" if the vehicle remained off the road in this way for $>15 \mathrm{~s}$. In the event of a "crash" the analysis program terminated at this point and subsequent analysis excluded these last $15 \mathrm{~s}$ to avoid overestimation of overall tracking error.

\section{Study design}

The 12 control subjects and 12 OSA patients had a period of training lasting $15 \mathrm{~min}$, based on the results of the study looking at the "learning effect". Each subject then underwent three $30 \mathrm{~min}$ tests where the "all", "near" and "far" fields were displayed in random order.

For the study investigating the "learning effect", 30 healthy subjects each performed seven tests lasting 6 min each. For the first test, all the road was visible. Following this the subjects were then randomized to drive with either "all", "near" or "far" road fields visible for the six following tests. A break of 6 min was allowed between each test.

Reproducibility data on consecutive days were obtained on 14 healthy subjects who had a prior training period of at least $12 \mathrm{~min}$ as determined in the learning study. The initial 5 min of training was spent with "all" the road field visible and subjects were then randomized to view "all", "near", or "far" road fields and spent the remaining training time on that field. Subjects then performed a 30 min simulation test on the field to which they had been randomized. The training and 30 min test were repeated at the same time on the following day.

Reproducibility data at greater time intervals were obtained on 12 healthy subjects using the protocol above but with the test separarted by 3-140 days (median 61 days).

Table 1. - Control and obstructive sleep apnoea (OSA) patients and steering results

\begin{tabular}{|c|c|c|c|}
\hline & $\begin{array}{l}\text { Control } \\
\text { subjects }\end{array}$ & $\begin{array}{c}\text { Patients } \\
\text { with OSA }\end{array}$ & $\mathrm{p}$-value \\
\hline Age yrs & $49(38 / 62)$ & $48(37 / 63)$ & 0.9 \\
\hline$>4 \% S \mathrm{a}, \mathrm{O}_{2} \operatorname{dips} \cdot \mathrm{h}^{-1}$ & $0.8(0 / 2.9)$ & $41.1(16 / 61)$ & $<0.0001$ \\
\hline ESS & $6.5(2.6 / 9.9)$ & $16.5(12.6 / 20.8)$ & $<0.0001$ \\
\hline SD "all" road & $0.11(0.09 / 0.25)$ & $0.23(0.12 / 0.9)$ & 0.001 \\
\hline SD "near" road & $0.14(0.1 / 0.25)$ & $0.55(0.28 / 1.36)$ & $<0.0001$ \\
\hline $\begin{array}{l}\text { SD "far" road } \\
\text { Reaction time }\end{array}$ & $0.2(0.18 / 0.42)$ & $0.47(0.31 / 0.68)$ & $<0.0001$ \\
\hline $\begin{array}{l}\text { "all" road } \\
\text { Reaction time }\end{array}$ & $1.8(1.3 / 2.4)$ & $2.6(1.4 / 4.9)$ & 0.024 \\
\hline $\begin{array}{l}\text { "near" road } \\
\text { Reaction time }\end{array}$ & $1.9(1.3 / 3.2)$ & $3.2(1.9 / 9.3)$ & 0.002 \\
\hline $\begin{array}{l}\text { "far" road } \\
\text { Off road events p }\end{array}$ & $1.8(1.2 / 3.0)$ & $2.8(1.6 / 6.8)$ & 0.017 \\
\hline $\begin{array}{l}\text { Oft road events pe } \\
\text { hour "all" road } \\
\text { Off road events pe }\end{array}$ & $0.0(0.0 / 3.6)$ & $5.2(0.0 / 118.0)$ & 0.028 \\
\hline $\begin{array}{l}\text { hour "near" road } \\
\text { Off road events per }\end{array}$ & $0.0(0.0 / 17.0)$ & $27.0(13.0 / 211.0)$ & $<0.0001$ \\
\hline hour "far" road & $0.0(0.0 / 22.0)$ & $88.0(17.0 / 230.0)$ & $<0.0001$ \\
\hline
\end{tabular}

Data are presented as medians with 5 th/95th centiles in parentheses. $\mathrm{Sa}_{2} \mathrm{O}_{2}$ : arterial oxygen saturation; ESS: Epworth sleepiness scale.
Table 2. - Reproducibility results on consecutive days and median of 61 days separation

\begin{tabular}{lcccc}
\hline & $\begin{array}{c}\text { First test } \\
\text { mean } \pm \text { SD }\end{array}$ & $\begin{array}{c}\text { Second test } \\
\text { mean } \pm \mathrm{SD}\end{array}$ & $\begin{array}{c}\text { Difference } \\
\text { mean } \pm \mathrm{SD}\end{array}$ \\
\hline $\begin{array}{l}\text { Consecutive days } \\
\mathrm{n}=14\end{array}$ & $0.20 \pm 0.17$ & $0.17 \pm 0.11$ & $-0.04 \pm 0.07 \mathrm{NS}$ \\
$\begin{array}{l}\text { Separated by median } \\
61 \text { days } \mathrm{n}=12\end{array}$ & $0.13 \pm 0.05$ & $0.13 \pm 0.05$ & $-0.001 \pm 0.04 \mathrm{NS}$ \\
\hline
\end{tabular}

Reproducibility of tests on the steering simulator on two occasions, separated by a day, or three or more days. The bias is represented by the mean difference and the precision by the SD of this difference. NS: nonsignificant.

\section{Results}

All results presented for the patient and control study are median and 5th and 95th centiles and the significance of any differences was assessed by Wilcoxon rank sum tests (table 1). Results of the learning effect study were assessed by one way analysis of variance (ANOVA). Reproducibility results are presented as mean and SD to represent the bias and precision, and the significance of any differences was assessed by paired t-test (table 2 ). A p-value of $<0.05$ was taken to indicate significance in all studies.

\section{Patients and controls}

Steering error was significantly worse in the patients with OSA compared to control subjects on all of the three roads $(\mathrm{p}<0.001$ for all comparisons, fig. 2$)$. The difference between patients and control subjects was least when "all" the road was visible, and greatest when only the "near" road was shown: the differences in median steering error between patients and control subjects was 0.12 for "all", 0.26 for "far", and 0.41 for "near".

The reaction times to detect the target number were significantly worse in patients compared to control subjects. Patients had significantly longer reaction times on all

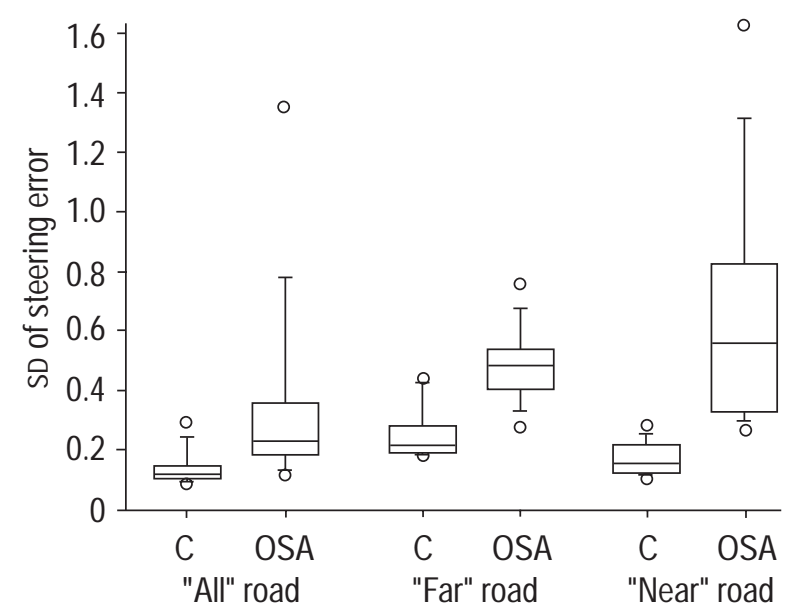

Fig. 2. - Steering performance, as assessed by the tendency to wander (SD of position, relative to road centre), in patients with obstructive sleep apnoea (OSA) and control subjects (C), when allowed to view the whole road ("all"), only the near field ("near"), or only the far field ("far"). Fifth, 25th, median, 75th and 95th centiles are shown. $\mathrm{p}<0.001$ for all comparisons (Wilcoxon). $\bigcirc$ : full range, i.e. maximum and minimum. 


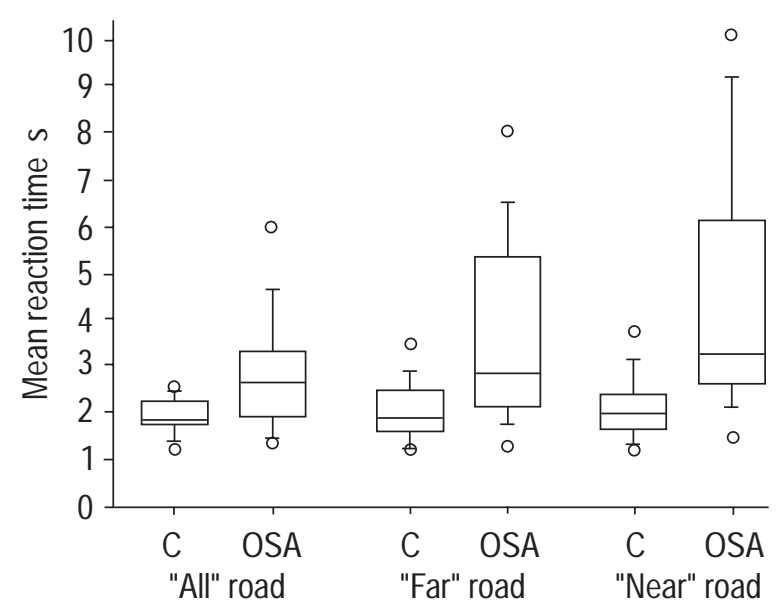

Fig. 3. - Reaction time during simulated steering assessed by the reaction time to the appearance of target numbers at the edges of the screen, in patients with obstructive sleep apnoea (OSA) and control subjects (C), when allowed to view the whole road ("all"), only the near field ("near"), or only the far field ("far"). Fifth, 25th, median, 75th and 95th centiles are shown. $\mathrm{p}<0.03$ for all comparisons (Wilcoxon). $\bigcirc$ : full range, i.e. maximum and minimum.

of the three roads when compared to the control group $(\mathrm{p}<0.03$; fig. 3). Patients had the longest reaction time when only the "near" road was displayed.

Patients also had significantly more "off road" events when compared with the control subjects on all ofthe three roads $(p<0.03$, fig. 4 ). Unlike the other measures of simulator performance, patients with OSA drove off the road most often when only the "far" road borders were visible.

\section{Learning effect study}

Results of each successive 5 min test are median and 5 th/95th centiles of steering error (fig. 5; only the randomised road tests 2 to 7 are represented in this figure). In this group of subjects the performance had reached a plateau

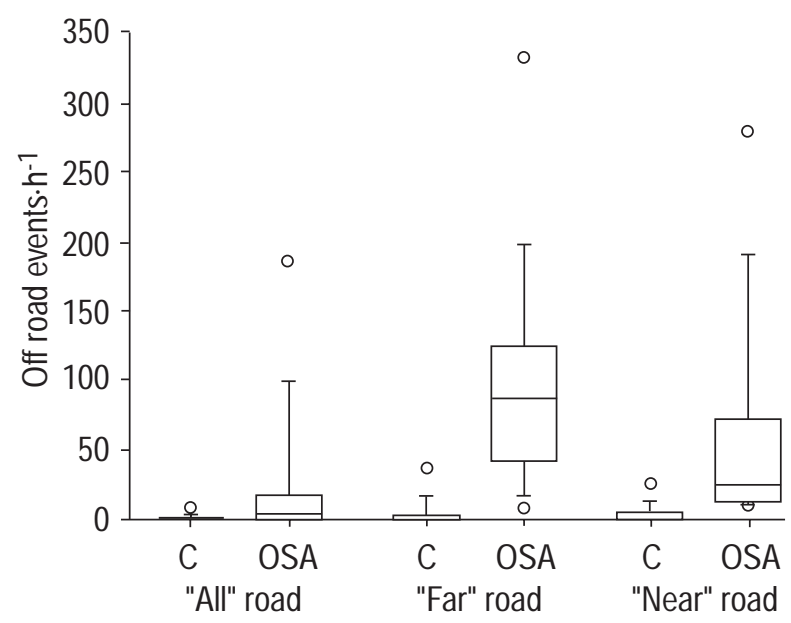

Fig. 4. - Steering performance, as assessed by the number of times per hour that the centre of the car bonnet crosses the edge of the road ("off road events"), in patients with obstructive sleep apnoea (OSA) and control subjects (C), when allowed to view the whole road ("all"), only the near field ("near"), or only the far field ("far"). Fifth, 25th, median, 75 th and 95 th centiles are shown. $\mathrm{p}<0.03$ for all comparisons (Wilcoxon). $\bigcirc$ : full range, i.e. maximum and minimum.

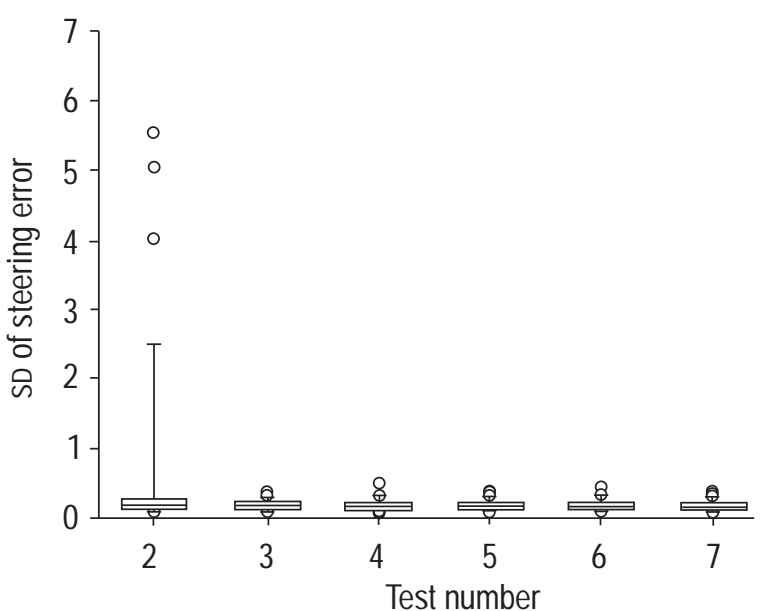

Fig. 5. - Steering performance, as assessed by the tendency to wander (sD of position, relative to road centre), across individual 6-min tests in healthy subjects, excluding the prerandomization test. Fifth, 25th, median, 75th and 95th centiles are shown. $\mathrm{p}<0.03$ for all comparisons (Wilcoxon). $\bigcirc$ : full range, i.e. maximum and minimum.

by $12 \mathrm{~min}$ of practice. One-way ANOVA confirmed that there was no significant difference between tests 3-7 and therefore a training period of at least 12 min was used in all subsequent subjects.

\section{Reproducibility studies}

Results are mean \pm SD and the significance of differences assessed by paired t-test. There were no significant differences between tests performed on consecutive days, or when separated by a longer time interval (table 2 ).

\section{Discussion}

The steering performance of OSA patients was significantly worse on all three simulation tests when compared with the matched control subjects. In addition they also had significantly longer reaction times when identifying target numbers at the periphery of the screen. The "learning effect" study suggests that only $12 \mathrm{~min}$ of practice is necessary before these simulation tests are run. Results from tests run on consecutive days at a set time, and also with a greater time interval between tests, were not significantly different.

Although "on the road" studies of driving performance are the only way to examine actual driving, there are a number of obvious reasons why they remain impractical. It is unethical to put frankly sleepy drivers behind the wheel of a motor vehicle and wait for them to make errors. The specific aspects of driving performance, which need to be measured to determine fitness to drive, remain unclear. Simple tests of vigilance may only be partially relevant to the complex tasks involved in driving a motor vehicle. In order to begin to study fitness to drive, it is necessary to analyse separate aspects of driving performance including steering accuracy, visual search and vigilance. Previous studies have examined some of these aspects of driving performance but none to date have used a realistic perspective view of the road ahead allowing the two visual 
aspects of steering a vehicle to be examined separately. In this study a group of patients with moderately severe OSA and excessive daytime sleepiness demonstrated significant impairment in all of the measures of driving performance relating to steering and visual search on a driving simulator, when compared with a group of matched control subjects.

The definition of sleep apnoea in this study was based on a simplified diagnostic system, the Visilab (Stowood Scientific Instruments), described in the "Methods" section. Quantifying OSA severity by counting $>4 \% \mathrm{Sa}_{\mathrm{a}} \mathrm{O}_{2}$ dips does not take into account the more subtle episodes of obstructed breathing that can occur without accompanying hypoxia. This approach will therefore tend to underestimate the degree of sleep fragmentation, but has been shown to be a valid technique in patients with this degree of OSA severity $[19,21]$.

The two study groups were all male and well matched for age, all had held driving licences for $>10 \mathrm{yrs}$, and drove $>250 \mathrm{~h} \cdot \mathrm{yr}^{-1}$. The authors did not match for other confounding factors that could conceivably influence the results that were obtained. Differential alcohol consumption by the two groups could be an important confounder. With the type of simulator used, proficiency or experience with video games could also have an influence. Visual problems are probably less important, since both patients and healthy subjects were free to use spectacles. Although the authors did not rigorously control for these potentially confounding variables, there is no reason to suspect that they affected one group more than the other. The most obvious difference between the groups was the presence or absence of OSA.

A number of authors have previously demonstrated impairment of simulated "driving" performance in patients with OSA [11, 16-18] and there is an increased risk of motor vehicle accidents in OSA patients [2-13]. The authors have confirmed impairment during all of the simulated driving conditions that they have examined. The authors found poorer reaction times in patients with OSA compared to healthy control subjects, although this was not the case in a recent study using a steering and divided attention task in patients with excessive sleepiness, including that due to OSA [18]. This previous simulator displays a box and a cross on the screen and uses a steering wheel to perform tracking correction to keep the cross within the box when subjected to random sideways forces. Thus it does not allow forward prediction of road curvature and is more akin to driving in fog or on a straight motorway with varying side winds. The tracking error was similarly quantified by the SD but the absolute values are not comparable with the current simulator. While tracking, the subject is also required to perform a visual search to recognize a target number appearing randomly in a corner of the screen, as in the simulator used in this study.

The authors have also shown in patients with OSA, but hardly at all in healthy subjects, that this impairment is exaggerated significantly when the opportunity to use one or other of the visual elements required for steering is removed. This is particularly the case for steering and reaction time when the distant curve of the road ahead is not visible, as would occur when driving in fog for example. This suggests that patients may be particularly dependent on the ability to predict the road ahead and thus became substantially worse when this element is removed. This may be because they have brief lapses in concentration or alertness that to some extent can be covered or compensated for when given access to advance information on the road curvature ahead, this strategy not being available of course when only the "near" field is visible.

When all of the road was visible, there was overlap of steering error between groups. This has been noted previously in a group of sleepy patients [17]. Therefore not all patients with excessive daytime sleepiness were worse than healthy subjects on the simulation. However when only the "near" field was visible, the best performing patient (driving error 0.25 ) only just reached the level of performance of the worst individual in the group of healthy subjects (driving error 0.27). Although the authors do not know how performance on the simulation relates to driving on a real road, this data suggests that the impaired steering ability of drivers with OSA could make them particularly impaired at driving under certain road conditions, especially when full information is denied them. No data are available on increased accident risk under specific road conditions, but clearly if this finding is confirmed then it would suggest that patients with OSA may be particularly at risk, not just in low stimulation conditions, but also in poor visibility conditions where full information about the road ahead is not available.

Thus, in conclusion, this steering simulator, with its more realistic view of the road ahead, has identified impaired performance in patients with obstructive sleep apnoea. In addition it has suggested that patients with obstructive sleep apnoea may be more disadvantaged than healthy subjects when the view of the road ahead is limited (such as in fog).

Acknowledgements. The authors would like to acknowledge the help of $\mathrm{M}$. Land in setting up the simulator.

\section{References}

1. Land M, Horwood J. Which parts of the road guide steering? Nature 1995; 377: 339-340.

2. Teran-Santos J, Jimenez-Gomez A, Cordero-Guevara J, the Cooperative Group Burgos-Santander. The association between sleep apnoea and the risk of traffic accidents. $N$ Engl J Med 1999; 340: 847-851.

3. George CF, Nickerson PW, Hanly PJ, Millar TW, Kryger MH. Sleep apnoea patients have more automobile accidents. Lancet 1987; 2: 447.

4. Findley LJ, Unverzagt ME, Suratt PM. Automobile accidents in patients with obstructive sleep apnea. Am Rev Respir Dis 1988; 138: 337-340.

5. Aldrich MS. Automobile accidents in patients with sleep disorders. Sleep 1989; 12: 487-494.

6. Haraldsson PO, Carenfelt C, Diderichsen F, Nygren A, Tingvall C. Clinical symptoms of sleep apnea syndrome and automobile accidents. J Otorhinolaryngol Relat Spec 1990; 52: 57-62.

7. Wu H, Yan Go F. Self-reported automobile accidents involving patients with obstructive sleep apnea. Neurology 1996; 46: 1254-1257.

8. Cassel W, Ploch T, Becker C, Dugnus D, Peter JH, von Wichert P. Risk of traffic accidents in patients with sleepdisordered breathing: reduction with nasal CPAP. Eur Respir J 1996; 9: 2606-2611. 
9. Findley LJ, Weiss JW, Jabour ER. Drivers with untreated sleep apnea. A cause of death and serious injury. Arch Intern Med 1991; 151: 1451-1452.

10. Findley LJ, Fabrizio M, Thommi G, Suratt PM. Severity of sleep apnea and automobile crashes. $N$ Engl J Med 1989; 320: 868-869.

11. Barbe F, Pericas J, Munoz A, Findley L, Anto JM, Agusti AG. Automobile accidents in patients with sleep apnea syndrome. An epidemiological and mechanistic study. Am J Respir Crit Care Med 1998; 158: 18-22.

12. Krieger J, Meslier N, Lebrun $\mathrm{T}$, et al. Accidents in obstructive sleep apnea patients treated with nasal continuous positive airway pressure: a prospective study. The Working Group ANTADIR, Paris and CRESGE, Lille, France. Association Nationale de Traitement a Domicile des Insuffisants Respiratoires. Chest 1997; 112: 15611566.

13. Engleman HM, Hirst WS, Douglas NJ. Under reporting of sleepiness and driving impairment in patients with sleep apnoea/hypopnoea syndrome. J Sleep Res 1997; 6: 272-275.

14. Engleman HM, Cheshire KE, Deary IJ, Douglas NJ. Daytime sleepiness, cognitive performance and mood after continuous positive airway pressure for the sleep apnoea/hypopnoea syndrome. Thorax 1993; 48: 911-914.
15. Naegele B, Thouvard V, Pepin JL, et al. Deficits of cognitive executive functions in patients with sleep apnea syndrome. Sleep 1995; 18: 43-52.

16. George CFP, Boudreau AC, Smiley A. Effects of nasal CPAP on simulated driving performance in patients with obstructive sleep apnoea. Thorax 1997; 52: 648-653.

17. Findley LJ, Fabrizio MJ, Knight H, Norcross BB, LaForte AJ, Suratt PM. Driving simulator performance in patients with sleep apnea. Am Rev Respir Dis 1989; 140: 529-530.

18. George CF, Boudreau AC, Smiley A. Simulated driving performance in patients with obstructive sleep apnea. $\mathrm{Am}$ J Respir Crit Care Med 1996; 154: 175-181.

19. Bennett LS, Langford BA, Stradling JR, Davies RJO. Sleep fragmentation indices as predictors of daytime sleepiness and nCPAP response in OSA. Am J Respir Crit Care Med 1998; 158: 778-786.

20. Pitson DJ, Sandell A, van den Hout R, Stradling JR. Use of pulse transit time as a measure of inspiratory effort in patients with obstructive sleep apnoea. Eur Respir J 1995; 8: 1669-1674.

21. Pitson DJ, Stradling JR. Autonomic markers of arousal during sleep in patients undergoing investigation for obstructive sleep apnoea, their relationship to EEG arousals, respiratory events and subjective sleepiness. $J$ Sleep Res 1998; 7: 53-60. 